\title{
Red Temática de Grupos: patología molecular de los tumores sólidos pediátricos
}

El cáncer infantil representa tan solo el 2\% de todos los cánceres. Pese a su prevalencia, el cáncer infantil ha sido el paradigma del desarrollo del tratamiento multidisciplinar en el contexto de grupos cooperativos tanto en el ámbito nacional como internacional, y en la actualidad más del 70\% de los niños diagnosticados de cáncer se curan de su proceso.

España cuenta desde el año 1980 con una herramienta de valor incalculable en el ámbito de la epidemiología del cáncer infantil, se trata del Registro Nacional de Tumores Infantiles - Sociedad Española de Oncología Pediátrica (RNTI-SEOP) ${ }^{1}$. A través del RNTISEOP se conoce la incidencia, supervivencia y mortalidad del cáncer infantil y por tanto permite valorar la calidad de nuestra asistencia y planificar la misma. La incidencia anual del cáncer infantil en España es de 132 nuevos casos por cada millón de niños de edad inferior a los 15 años. Actualmente la tasa de supervivencia global de los niños afectados de cáncer es del $73 \%$, ello supone 19 puntos de aumento con relación a la supervivencia obtenida en los pacientes que fueron diagnosticados en el trienio 1980-1982, así como una disminución del 45\% en el riesgo de muerte en los cinco años posteriores al diagnóstico de cáncer. Este avance ha sido posible fundamentalmente gracias a la quimiosensibilidad de las neoplasias pediátricas - la mayoría de las cuales a diferencia de lo que ocurre en el adulto no son de origen epitelial -, a los nuevos conceptos quirúrgicos y a una redefinición de las indicaciones de la radioterapia. A ello hay que añadir un mejor conocimiento biológico de los tumores de la infancia, lo cual en la actualidad incide en la supervivencia. Sin embargo, a pesar de los avances, el cáncer sigue siendo la segunda causa de mortalidad infantil en los países desarrollados, a la vez que puede generar efectos secundarios en los supervivientes, los cuales deben de intentar prevenirse y minimizar. Del conjunto de neoplasias infantiles se identifican subgrupos de alto riesgo que no comparten los satisfactorios resultados, se trata de procesos linfoproliferativos como la leucemia mieloide aguda y la leucemia linfoblástica aguda con anomalías cromosómicas $\mathrm{t}(9 ; 22)$ o $\mathrm{t}(4 ; 11)$ al igual que tumores sólidos como el neuroblastoma con biología desfavorable, los gliomas de tronco o de alto riesgo de otras localizaciones, el meduloblastoma de riesgo elevado y los sarcomas óseos o de partes blandas.

Si bien el cáncer es el resultado de diversas alteraciones genéticas su causa en la mayoría de ellos es desconocida. En el cáncer pediátrico existen casos de predisposición genética la cual puede ser familiar, como en el retinoblastoma o en el síndrome de LiFraumeni. También poblaciones pediátricas portadoras de anomalías cromosómicas como la trisomía 21 o de malformaciones congénitas tales como la aniridia o la hemihipertrofia presentan mayor riesgo de cáncer. Sin embargo, la mayoría de los cánceres pediátricos ocurren de forma esporádica y no se hallan asociados a mutaciones germinales o anomalías congénitas. Virus, como el de Epstein-Barr o el de la hepatitis B se han involucrado en la etiología de algunos cánceres. Ciertos fármacos de uso terapéutico como los inhibidores de la topoisomerasa 2, los agentes alquilantes y la irradiación terapéutica pueden ser inductores de segundas neoplasias.

La mayoría de los tumores sólidos infantiles comparten peculiaridades histológicas a la microscopia óptica como son la de hallarse constituidos por células pequeñas y redondas lo cual no pocas veces comportaba dificultades diagnósticas. En la actualidad las nuevas tecnologías, inmunohistoquímica, microscopia electrónica y las técnicas moleculares no solo están aportando luz al correcto diagnóstico sino que también desarrollan un papel fundamental en el pronóstico, seguimiento y definición del tratamiento de las neoplasias infantiles. Todo ello ha llevado a una reconsideración de las características de la cantidad y calidad del material biópsico a obtener en el proceso diagnóstico. El material conseguido en fresco debe de ser suficiente para su estudio por microscopia óptica e inmunohistoquímica, microscopia electrónica, biología molecular, estudio cromosómico, FISH y citometría de flujo.

Si bien los avances en el tratamiento del cáncer infantil se han basado en el tratamiento multidisciplinar en el contexto de unidades oncológicas pediátricas, el desarrollo de la investigación científica aplicada al cáncer infantil fue desde sus inicios fruto del esfuerzo individual de científicos que compaginaban su quehacer clínico diario con la investigación aplicada. El 
Nodos de la red

Nodo 1. Grupo de Oncología Molecular en Tumores Pediátricos. Hospital Materno-Infantil Vall d’Hebron. Barcelona.

Coordinador: José Sánchez de Toledo Codina.

Responsable Patología Molecular: Soledad Gallego Melcon.

Nodo 2. Grupo para el estudio molecular de Tumores sólidos infantiles.

Hospital Sant Joan de Deu. Barcelona.

Coordinador: Luis Morales Fochs.

Responsable Patología Molecular: Jaume Mora Graupera.

Nodo 3. Grupo de Trabajo de Tumores cerebrales infantiles.

Hospital de Cruces. Baracaldo.

Coordinador: Aurora Navajas Gutiérrez.

Nodo 4. Grupo de Genética Molecular del Cáncer. Instituto de Investigaciones Biomédicas - Unidad Oncohematología, Hospital Materno-Infantil La Paz. Madrid.

Coordinador : Ángel Pestaña Vargas.

Responsable clínico: Purificación García Miguel.

Nodo 5. Servicio de Onco-hematología

Hospital Universitario Niño Jesús. Madrid

Coordinador: Manuel Ramírez Orellana

Nodo 6. Grupo de Patología Molecular de tumores sólidos infantiles.

Centro de Investigación del Cáncer. Universidad de SalamancaCSIC. Salamanca

Coordinador: Enrique de Alava Casado

Nodo 7. Grupo de Oncología Molecular en Pediatría Hospital Universitario Virgen del Rocio. Sevilla

Coordinador : Dolores Segura Ayestaran

Nodo 8. Departamento de Patología

Facultad de Medicina. Universidad de Valencia. Valencia

Coordinador: Samuel Navarro Fos

Nodo 9. Unidades de Oncología Pediátrica y Genética

Hospital Universitario La Fe. Valencia

Coordinador : Victoria Castel Sánchez

Nodo 10. Registro Nacional de Tumores Infantiles

Instituto López Piñero CSIC-UV. Valencia

Coordinador: Rafael Peris Bonet

desarrollo de ensayos cooperativos nacionales e internacionales conllevó que de forma progresiva fueran incluidos en ellos aquellos estudios concernientes a la biología molecular que podían tener impacto en el diagnóstico, pronóstico, seguimiento y en la individualización del tratamiento y por tanto en el incremento de la supervivencia.

La comunidad científica pediátrica española dedicada a la oncología ha desarrollado en los últimos años un entramado de colaboración, integrada en los diversos estudios cooperativos auspiciados por la Sociedad Española de Oncología Pediátrica y la Sociedad Internacional de Oncología Pediátrica, que dieron sus frutos en el campo de la biología molecular del neuroblasto- ma, del sarcoma de Ewing y de los sarcomas de partes blandas, contribuyendo al diagnóstico, definiendo el pronóstico y ayudando a individualizar el tratamiento. Todo ello ha sido el germen de la recientemente creada Red para la Investigación de los Tumores Sólidos Infantiles.

La Red para la Investigación de los Tumores Sólidos Infantiles $^{2}$ (RITSI), es la Red Temática de nodos, financiada por el Instituto de Salud Carlos III, correspondiente al Proyecto " Patología molecular de los tumores sólidos infantiles: aportación al diagnóstico y a la individualización del tratamiento". La Red ha sido fruto de la necesidad de establecer un programa de investigación que integrando diversos grupos permita una mejor adecuación de los recursos existentes tanto científicos como tecnológicos en aras de progresar en el conocimiento del cáncer infantil y contribuir a incrementar su supervivencia. Este centro virtual de investigación en Oncología pediátrica actualmente se basa en diez hospitales y centros públicos de investigación localizados en diferentes Comunidades Autónomas (Andalucía, Castilla- León, Cataluña, Comunidad Valenciana, Madrid y País Vasco), los cuales se agrupan en un proyecto que comprende el desarrollo de cinco programas y cuatro líneas de investigación traslacional. Todos ellos dirigidos al estudio fundamentalmente de patologías oncológicas pediátricas como son el neuroblastoma, rabdomiosarcoma, meduloblastoma y sarcoma de Ewing, en su fase inicial. El programa de formación en patología molecular en tumores sólidos, el soporte epidemiológico del RNTI, la creación de un Banco de tumores sólidos infantiles, la armonización de técnicas de diagnóstico y detección de enfermedad residual mínima y la aplicación de metodologías genómicas de alto rendimiento con la finalidad de clasificar y individualizar el tratamiento han de llevar a lograr los fines de la red.

Se ha creado la pagina web de la RITSI (http://ritsi.uv.es) como órgano de información y gestión de las diversas actividades de la Red. La elaboración del documento de consenso sobre " aspectos y recomendaciones sobre el funcionamiento de los bancos de tumores integrados en la red" ha permitido progresar en la puesta en marcha de los bancos de tumores de los centros integrados en la red. Se han armonizado y validado las diferentes técnicas de diagnóstico molecular en los tumores de la familia del Ewing, rabdomiosarcoma, tumor desmoplásico y sarcoma sinovial y de los estudios de enfermedad mínima residual en los tumores de la familia del Ewing, rabdomiosarcoma alveolar y neuroblastoma. La identificación de marcadores de progresión y de respuesta al tratamiento está siendo también motivo de estudio, así como el diseño de microarray específicos para la caracterización de alteraciones genéticas en los tumores sólidos. 
La Red se pone a disposición de la comunidad científica para progresar conjuntamente en el conocimiento del cáncer infantil y avanzar en el objetivo común de incrementar la supervivencia de los niños afectos de cáncer.

\section{Bibliografía}

1. Peris-Bonet R, et al. RNTI-SEOP Estadísticas Básicas 1980-2001.Supervivencia 1980-1997.Ed. Universidad de Valencia, 2003.
2. RITSI. Red de Investigación de Tumores sólidos infantiles http://ritsi.uv.es

José Sánchez de Toledo Codina Coordinador de la Red de Investigación en Tumores Sólidos Infantiles. Servicio de Oncología y Hematología Pediátrica. Hospital Materno-Infantil Vall d'Hebron. Barcelona 